\title{
Bootstrap Approach to the One-Sample and Two-Sample Test of Variances of a Fuzzy Random Variable
}

\author{
Jalal Chachi* \\ Department of Mathematics, Statistics and Computer Sciences, Semnan University, Iran
}

(Received: 11 January 2017; Accepted: 14 August 2017)

\begin{abstract}
The aim of this paper is to present in a concise and integrated way of the bootstrap approach to statistical testing of hypotheses about the variance of fuzzy random variable. In this approach, first a notion of fuzzy random variables is recalled. Then, we will consider hypothesis-tests for the (crisp-valued) variance of fuzzy data in a population. For this purpose, the $\alpha$-pessimistic values of the imprecise observations are used for defining a new notion of distance measure between fuzzy data, which is then used to make a procedure for testing the statistical hypotheses. Based on this argument, the application of bootstrap techniques in dealing with these testing problems will be introduced. The procedure develops a non-parametric approach to testing statistical hypotheses based on one-sample and two-sample fuzzy data.
\end{abstract}

Keywords $\alpha$-Pessimistic, Bootstrap, Fuzzy Random Variable, Testing Hypotheses

AMS 2010 subject classifications 94D05; 62G08; 62J86

DOI: $10.19139 /$ soic.v5i3.267

\section{Introduction}

The bootstrap techniques contained in the literature are simple and straight-forward methods for calculating approximated biases, standard deviations, confidence intervals, and so forth, in almost any non-parametric estimation problem $[8,31]$. As a common conclusion, we can state that bootstrap techniques are very valuable in dealing with testing about means and variances of imprecise concepts such as fuzzy random variables, since

1. there are not stochastic models in the literature which can be really widely applicable to describe imprecise quantities involved in practical situations,

2. most of the parameters in general asymptotic approaches could be unknown, the accuracy of bootstrap approaches is greater than that of asymptotic ones.

On the other hand, researchers in economics, engineering, environment science, the social science, medical science, business, management, and many other fields deal daily with the complexities of modeling uncertain data. Classical methods are not always successful dealing with uncertain data, because the uncertainties appearing in these domains may be of various types, such as probability theory [4], fuzzy set theory [36], intuitionistic fuzzy set theory [2], vague set theory [10], interval mathematics [14], and other mathematical tools are well know and often useful approaches to describing uncertainty. Therefore, in the studies proposed by Montenegro et al. [25], González-Rodríguez et al. [13], and Gil et al. [11] it has been stated that the well-known bootstrap techniques are a valuable tool in testing statistical hypotheses about the means and variances of fuzzy random variables, when these

\footnotetext{
${ }^{*}$ Correspondence to: Jalal Chachi (Email: jchachi@ semnan.ac.ir). Department of Mathematics, Statistics and Computer Sciences, Semnan
} University, Semnan 35195-363, Iran.

ISSN 2310-5070 (online) ISSN 2311-004X (print)

Copyright (C) 2017 International Academic Press 
variables are supposed to take on a finite number of different values and these values being fuzzy subsets of the one-dimensional Euclidean space (see also, [1, 7, 32, 37, 35]).

Therefore, the proposed paper provides the bootstrap approach to statistical testing of hypotheses about the variance of fuzzy random variable. For this purpose, the $\alpha$-pessimistic values of the imprecise observations are used for defining the new notion of fuzzy random variables as well as a new notion of distance measure between fuzzy data. The procedure develops a non-parametric approach to testing statistical hypotheses based on one-sample and two-sample fuzzy data.

The paper is organized as follows: In the next section, some basic concepts of fuzzy set theory and uncertainty theory are recalled. In this section also a new definition of distance measure between fuzzy numbers is defined. In Section 3, a new definition of fuzzy random variable proposed by Hesamian and Chachi [15] is recalled. In Section 4, the non-parametric approach to testing statistical hypotheses based on one-sample and two-sample fuzzy data is provided. Numerical examples are also given in this section to clarify the proposed testing method. Finally, a brief conclusion is provided in Section 5.

\section{Preliminary concepts}

In this section, first, we shall review the basic definitions and terminologies of the fuzzy set theory and uncertainty theory which are necessary for our paper (for further details, the reader is referred to [21, 22, 33, 38]). Then, a new definition of distance measure between fuzzy numbers is defined.

A fuzzy set $\widetilde{A}$ of the universal set $\mathbb{X}$ is defined by its membership function $\widetilde{A}: \mathbb{X} \rightarrow[0,1]$. In this paper, we consider $\mathbb{R}$ (the real line) as the universal set. We denote by $\widetilde{A}[\alpha]=\{x \in \mathbb{R}: \widetilde{A}(x) \geq \alpha\}$ the $\alpha$-level set ( $\alpha$-cut) of the fuzzy set $\widetilde{A}$ of $\mathbb{R}$, for every $\alpha \in(0,1]$, and $\widetilde{A}[0]$ is the closure of the set $\{x \in \mathbb{R}: \widetilde{A}(x)>0\}$. A fuzzy set $\widetilde{A}$ of $\mathbb{R}$ is called a fuzzy number if for every $\alpha \in[0,1]$, the set $\widetilde{A}[\alpha]$ is a non-empty compact interval. We denote by $\mathcal{F}(\mathbb{R})$, the set of all fuzzy numbers of $\mathbb{R}$.

The imprecision or vagueness can be treated by means of a particular kind (family) of fuzzy numbers, the $L R$ fuzzy numbers. These are very useful in practice since they can be characterized by means of three real numbers: the center, the left spread, and the right spread. The term $L R$ is due to the left $(L)$ and the right $(R)$ shape of the membership function referred to the fuzzy set. Typically, the $L R$ fuzzy number $\widetilde{N}=(n, l, r)_{L R}$ with central value $n \in \mathbb{R}$, left and right spreads $l \in \mathbb{R}^{+}, r \in \mathbb{R}^{+}$, decreasing left and right shape functions $L: \mathbb{R}^{+} \rightarrow[0,1]$, $R: \mathbb{R}^{+} \rightarrow[0,1]$, with $L(0)=R(0)=1$, has the following membership function

$$
\widetilde{N}(x)=\left\{\begin{array}{lll}
L\left(\frac{n-x}{l}\right) & \text { if } \quad x \leq n, \\
R\left(\frac{x-n}{r}\right) & \text { if } \quad x \geq n .
\end{array}\right.
$$

We can easily obtain the $\alpha$-cut of $\tilde{N}$ as follows

$$
\tilde{N}[\alpha]=\left[n-L^{-1}(\alpha) l, n+R^{-1}(\alpha) r\right], \quad \alpha \in[0,1] .
$$

A special type of $L R$-fuzzy numbers is the so-called triangular fuzzy numbers. The membership function and the $\alpha$-cut of triangular fuzzy number $\widetilde{A}=(a, l, r)_{T}$ is given by

$$
\begin{aligned}
& \widetilde{A}(x)=\left\{\begin{array}{lrl}
0 & x & <a-l, \\
\frac{x-a+l}{l} & a-l \leq x & <a, \\
\frac{a+r-x}{r} & a \leq x & \leq a+r, \\
0 & x & >a+r .
\end{array}\right. \\
& \widetilde{A}[\alpha]=[n-l(1-\alpha), n+r(1-\alpha)], \quad \alpha \in[0,1] .
\end{aligned}
$$

For the algebraic operations of $L R$-fuzzy numbers, we have the following result on the basis of Zadeh's extension principle. Let $\widetilde{M}=\left(m, l_{m}, r_{m}\right)_{L R}$ and $\widetilde{N}=\left(n, l_{n}, r_{n}\right)_{L R}$ be two triangular fuzzy numbers and $\lambda$ be a real number. 
Then

$$
\begin{aligned}
\lambda \otimes \widetilde{M} & =\left\{\begin{array}{lll}
\left(\lambda m, \lambda l_{m}, \lambda r_{m}\right)_{L R} & \text { if } & \lambda>0, \\
\mathcal{I}_{\{0\}} & \text { if } & \lambda=0, \\
\left(\lambda m,|\lambda| r_{m},|\lambda| l_{m}\right)_{R L} & \text { if } & \lambda<0,
\end{array}\right. \\
\widetilde{M} \oplus \widetilde{N} & =\left(m+n, l_{m}+l_{n}, r_{m}+r_{n}\right)_{L R},
\end{aligned}
$$

where $\mathcal{I}_{\{0\}}$ stands for the indicator function of the crisp zero.

In the following, we introduce an index to compare fuzzy number $\widetilde{A} \in \mathcal{F}(\mathbb{R})$ and crisp value $x \in \mathbb{R}$. The index will be used for defining a new notion of fuzzy random variable.

Definition 1 ([23])

Let $\widetilde{A} \in \mathcal{F}(\mathbb{R})$ and $x \in \mathbb{R}$. The index $C: \mathcal{F}(\mathbb{R}) \times \mathbb{R} \longrightarrow[0,1]$, which is defined by

$$
C\{\widetilde{A} \leq x\}=\frac{\sup _{y \leq x} \widetilde{A}(y)+1-\sup _{y>x} \widetilde{A}(y)}{2}
$$

shows the credibility degree that $\widetilde{A}$ is less than or equal to $x$. Similarly, $C\{\widetilde{A}>x\}=1-C\{\widetilde{A} \leq x\}$ shows the credibility degree that $\widetilde{A}$ is greater than $x$ (see also [22]).

Definition 2

Let $\widetilde{A} \in \mathcal{F}(\mathbb{R})$ and $\alpha \in[0,1]$, then $\widetilde{A}_{\alpha}=\inf \{x \in \widetilde{A}[0]: C\{\widetilde{A} \leq x\} \geq \alpha\}$, is called the $\alpha$-pessimistic value of $\widetilde{A}$. It is clear that $\widetilde{A}_{\alpha}$ is a non-decreasing function of $\alpha \in(0,1]$ (for more details, see [21, 26]).

Example 1

Suppose that $\widetilde{A}=(a, l, r)_{L R}$ is a $L R$-fuzzy number, and let $x \in \mathbb{R}$, then

$$
C\{\widetilde{A} \leq x\}= \begin{cases}\frac{1}{2} L\left(\frac{a-x}{l}\right) & \text { if } \quad x \leq a, \\ 1-\frac{1}{2} R\left(\frac{x-a}{r}\right) & \text { if } \quad x \geq a .\end{cases}
$$

We can easily obtain the $\alpha$-pessimistic values of $\widetilde{A}$ as follows

$$
\widetilde{A}_{\alpha}= \begin{cases}a-l L^{-1}(2 \alpha) & \text { for } \quad 0.0<\alpha \leq 0.5 \\ a+r R^{-1}(2(1-\alpha)) & \text { for } \quad 0.5 \leq \alpha \leq 1.0\end{cases}
$$

In the following, based on the notion of $\alpha$-pessimistic value of fuzzy sets, a new definition of metrics between fuzzy numbers is defined. In the literature one can find many useful metrics between fuzzy numbers and a few ones between fuzzy values. Valuable references on this point can be found in [5].

\section{Definition 3}

The distance measure is defined as the mapping $D: \mathcal{F}(\mathbb{R}) \otimes \mathcal{F}(\mathbb{R}) \rightarrow[0, \infty)$ such that it associates with two fuzzy numbers $\widetilde{A}, \widetilde{B} \in \mathcal{F}(\mathbb{R})$ the value $D(\widetilde{A}, \widetilde{B})$ such that

$$
D(\widetilde{A}, \widetilde{B})=\int_{0}^{1}\left(\widetilde{A}_{\alpha}-\widetilde{B}_{\alpha}\right)^{2} d \alpha .
$$

One can conclude that the mapping $D: \mathcal{F}(\mathbb{R}) \otimes \mathcal{F}(\mathbb{R}) \rightarrow[0, \infty)$ satisfies the following conditions

1. For any $\widetilde{A}, \widetilde{B} \in \mathcal{F}(\mathbb{R}), D(\widetilde{A}, \widetilde{B})=0$ if and only if $\widetilde{A}=\widetilde{B}$.

2. For any $\widetilde{A}, \widetilde{B} \in \mathcal{F}(\mathbb{R}), D(\widetilde{A}, \widetilde{B})=D(\widetilde{B}, \widetilde{A})$.

3. For any $\widetilde{A}, \widetilde{B}, \widetilde{C} \in \mathcal{F}(\mathbb{R}), D(\widetilde{A}, \widetilde{C}) \leq D(\widetilde{A}, \widetilde{B})+D(\widetilde{B}, \widetilde{C})$.

4. For any $\widetilde{A}, \widetilde{B}, \widetilde{C} \in \mathcal{F}(\mathbb{R})$, such that $\widetilde{A} \subseteq \widetilde{B} \subseteq \widetilde{C}$, then $D(\widetilde{A}, \widetilde{C}) \geq \max \{D(\widetilde{A}, \widetilde{B}), D(\widetilde{B}, \widetilde{C})\}$. 
As an example, we can easily obtain the distance between two $L R$-fuzzy numbers $\widetilde{A}=\left(a, l_{1}, r_{1}\right)_{L R}$ and $\widetilde{B}=\left(b, l_{2}, r_{2}\right)_{L R}$ as follows

$$
\begin{aligned}
D(\widetilde{A}, \widetilde{B})= & (a-b)^{2}+\frac{\left(l_{1}-l_{2}\right)^{2}}{2} \int_{0}^{1}\left(L^{-1}(\alpha)\right)^{2} d \alpha+\frac{\left(r_{1}-r_{2}\right)^{2}}{2} \int_{0}^{1}\left(R^{-1}(\alpha)\right)^{2} d \alpha \\
& -(a-b)\left(l_{1}-l_{2}\right) \int_{0}^{1} L^{-1}(\alpha) d \alpha+(a-b)\left(r_{1}-r_{2}\right) \int_{0}^{1} R^{-1}(\alpha) d \alpha .
\end{aligned}
$$

For symmetric fuzzy numbers $\widetilde{A}=(a, l, l)_{L}$ and $\widetilde{B}=(b, r, r)_{L}$ we have

$$
D(\widetilde{A}, \widetilde{B})=(a-b)^{2}+(l-r)^{2} \int_{0}^{1}\left(L^{-1}(\alpha)\right)^{2} d \alpha .
$$

\section{Fuzzy random variables}

In the context of random experiments whose outcomes are not numbers (or vectors in $\mathbb{R}^{p}$ ) but they are expressed in inexact terms, the concept of fuzzy random variable turns out to be useful. Random fuzzy numbers (or, more generally, random fuzzy sets [5]) is a well-stated and supported model within the probabilistic setting for the random mechanisms generating fuzzy data. They integrate randomness and fuzziness, so that the first one affects the generation of experimental data, whereas the second one affects the nature of experimental data which are assumed to be intrinsically imprecise. The notion of random fuzzy set can be formalized in several equivalent ways. Thus, in this regard, different notions of fuzzy random variable have been introduced and investigated from different points of view in the literature, e.g. see, [5, 6, 12, 15, 17, 18, 19, 20, 24, 27, 28, 29, 30].

Definition 4 ([15])

Suppose that a random experiment is described by a probability space $(\Omega, \mathcal{A}, \mathbf{P})$, where $\Omega$ is a set of all possible outcomes of the experiment, $\mathcal{A}$ is a $\sigma$-algebra of subsets of $\Omega$ and $\mathbf{P}$ is a probability measure on the measurable space $(\Omega, \mathcal{A})$. The fuzzy-valued mapping $\widetilde{X}: \Omega \rightarrow \mathcal{F}(\mathbb{R})$ is called a fuzzy random variable if for any $\alpha \in[0,1]$, the real-valued mapping $\widetilde{X}_{\alpha}: \Omega \rightarrow \mathbb{R}$ is a real-valued random variable on $(\Omega, \mathcal{A}, \mathbf{P})$. Throughout this paper, we assume that all random variables have the same probability space $(\Omega, \mathcal{A}, \mathbf{P})$.

\section{Remark 1}

Kwakernaak $[19,20]$ introduced the notion of fuzzy random variables which has been later formalized in a clear way by Kruse and Meyer [18]. In this approach, the fuzzy random variable is viewed as a fuzzy perception (observation or report) of a classical real-valued random variable which is equivalent as: given a probability space $(\Omega, \mathcal{A}, \mathbf{P})$, a mapping $\widetilde{X}: \Omega \rightarrow \mathcal{F}(\mathbb{R})$ is said to be a fuzzy random variable if for all $\alpha \in(0,1]$ the two real-valued mappings $\widetilde{X}_{\alpha}^{L}: \Omega \rightarrow \mathbb{R}$ and $\widetilde{X}_{\alpha}^{U}: \Omega \rightarrow \mathbb{R}$ are real-valued random variables (see also $[6,27,28]$ ).

One can easily show that the following relations are also held between the definition of fuzzy random variable proposed in this paper and Kwakernaak and Kruse's definition (see also, Example 1)

$$
\begin{aligned}
\tilde{X}_{\alpha} & = \begin{cases}\widetilde{X}_{2 \alpha}^{L} & \text { for } 0.0<\alpha \leq 0.5, \\
\widetilde{X}_{2(1-\alpha)}^{U} & \text { for } 0.5 \leq \alpha \leq 1.0,\end{cases} \\
\widetilde{X}[\alpha] & =\left[\widetilde{X}_{\frac{\alpha}{2}}, \widetilde{X}_{1-\frac{\alpha}{2}}\right],
\end{aligned}
$$

The first above relation shows that the information contained in the two-dimensional variable $\left(\widetilde{X}_{\alpha}^{L}, \widetilde{X}_{\alpha}^{U}\right)$ is summarized in the one-dimensional variable $\widetilde{X}_{\alpha}$ making the computational procedures in the problems more easier. These relations confirms that this kind of definition of fuzzy random variables is equivalent to that of Kwakernaak and Kruse's definition.

Using classical techniques in Probability Theory, some aspects and results associated to a random variable were extended to this new environment (see e.g. [6, 15, 29, 30, 37]). 
Definition 5 ([15])

Two fuzzy random variables $\widetilde{X}$ and $\widetilde{Y}$ are said to be independent if $\widetilde{X}_{\alpha}$ and $\widetilde{Y}_{\alpha}$ are independent, for all $\alpha \in[0,1]$. In addition, we say that two fuzzy random variables $\widetilde{X}$ and $\widetilde{Y}$ are identically distributed if $\widetilde{X}_{\alpha}$ and $\widetilde{Y}_{\alpha}$ are identically distributed, for all $\alpha \in[0,1]$. Similar arguments can be used for more than two fuzzy random variables. We also say that $\widetilde{X}_{1}, \ldots, \widetilde{X}_{n}$ is a fuzzy random sample if $\widetilde{X}_{i}$ 's are independent and identically distributed fuzzy random variables. We denote by $\widetilde{x}_{1}, \ldots, \widetilde{x}_{n}$ the observed values of fuzzy random sample $\widetilde{X}_{1}, \ldots, \widetilde{X}_{n}$.

In analyzing fuzzy data two main types of summary measures/parameters may be distinguished:

1. fuzzy-valued summary measures, like the mean value of a fuzzy random variable or the median of a fuzzy random variable as measures for the central tendency of their distributions [3, 9, 34];

2. real-valued summary measures, like the variance of a fuzzy random variable as a measure for the mean error/dispersion of the distributions of the fuzzy random variable, or the covariance as a measure of the (absolute) linear dependence/association of a fuzzy random variable [6, 16, 29].

The above summary measures for the fuzzy random variable as well as some properties of them are formalized as follows:

Definition 6

Given a probability space $(\Omega, \mathcal{A}, \mathbf{P})$ and an associated fuzzy random variable $\widetilde{X}: \Omega \rightarrow \mathcal{F}(\mathbb{R})$ such that for any $\alpha \in[0,1]$, the real-valued variable $\widetilde{X}_{\alpha}: \Omega \rightarrow \mathbb{R}$ on $(\Omega, \mathcal{A}, \mathbf{P})$ has finite mean (i.e. $\widetilde{X}_{\alpha} \in L^{1}(\Omega, \mathcal{A}, \mathbf{P})$ ), the mean value of $\widetilde{X}$ is the fuzzy value $\widetilde{E}(\widetilde{X}) \in \mathcal{F}(\mathbb{R})$ such that for all $\alpha \in[0,1]$

$$
\widetilde{E}(\widetilde{X})_{\alpha}=E\left(\widetilde{X}_{\alpha}\right)=\int_{\Omega} \widetilde{X}_{\alpha} d \mathbf{P}
$$

\section{Proposition 1}

$\widetilde{E}$ is additive (i.e., equivariant under the sum of fuzzy random variables), that is, for fuzzy random variables $\widetilde{X}$ and $\widetilde{Y}$ associated with the same probability space $(\Omega, \mathcal{A}, \mathbf{P})$ and such that $\widetilde{X}_{\alpha}, \widetilde{Y}_{\alpha} \in L^{1}(\Omega, \mathcal{A}, \mathbf{P})$, we have that

$$
\begin{aligned}
\widetilde{E}(\widetilde{X} \oplus \widetilde{Y}) & =\widetilde{E}(\widetilde{X}) \oplus \widetilde{E}(\widetilde{Y}), \\
\widetilde{E}(\lambda \otimes \widetilde{X}) & =\lambda \otimes \widetilde{E}(\widetilde{X}), \quad \lambda \in \mathbb{R} .
\end{aligned}
$$

Definition 7

The variance of a fuzzy random variable $\widetilde{X}$ is defined as

$$
\begin{aligned}
\nu(\widetilde{X}) & =E[D(\widetilde{X}, \widetilde{E}(\widetilde{X}))] \\
& =E\left(\int_{0}^{1}\left(\widetilde{X}_{\alpha}-E\left(\widetilde{X}_{\alpha}\right)\right)^{2} d \alpha\right) \\
& =\int_{\Omega} \int_{0}^{1}\left(\widetilde{X}_{\alpha}-E\left(\widetilde{X}_{\alpha}\right)\right)^{2} d \alpha d \mathbf{P} \\
& =\int_{0}^{1} \int_{\Omega}\left(\widetilde{X}_{\alpha}-E\left(\widetilde{X}_{\alpha}\right)\right)^{2} d \mathbf{P} d \alpha \\
& =\int_{0}^{1} \operatorname{Var}\left(\widetilde{X}_{\alpha}\right) d \alpha .
\end{aligned}
$$

\section{Proposition 2}

Let $\widetilde{\mathbf{X}}=\left(\widetilde{X}_{1}, \widetilde{X}_{2}, \ldots, \widetilde{X}_{n}\right)$ be a fuzzy random sample, and $S_{n}^{2}(\widetilde{\mathbf{X}})=\frac{1}{n-1} \sum_{i=1}^{n} D\left(\widetilde{X}_{i}, \overline{\widetilde{\mathbf{X}}}\right)$ be the crisp variance value of the fuzzy sample $\widetilde{\mathbf{X}}$, where $\overline{\widetilde{\mathbf{X}}}=\frac{1}{n} \oplus_{i=1}^{n} \widetilde{X}_{i}$ is the fuzzy sample mean value. Then $S_{n}^{2}(\widetilde{\mathbf{X}})$ is an unbiased estimator of the parameter $\nu(\widetilde{X})$ (population variance), i.e. $E\left[S_{n}^{2}(\widetilde{\mathbf{X}})\right]=\nu(\widetilde{X})$; and $\lim _{n \rightarrow \infty} S_{n}^{2}(\widetilde{\mathbf{X}})=\nu(\widetilde{X})$. 
Proof

$$
\begin{aligned}
E\left[S_{n}^{2}\right] & =\frac{1}{n-1} \sum_{i=1}^{n} \int_{0}^{1} E\left[\widetilde{X}_{i \alpha}-\overline{\widetilde{\mathbf{X}}}_{\alpha}\right]^{2} d \alpha \\
& =\frac{1}{n-1} \sum_{i=1}^{n} \int_{0}^{1} E\left[\widetilde{X}_{i \alpha}-E\left(\widetilde{X}_{i \alpha}\right)+E\left(\widetilde{X}_{i \alpha}\right)-\overline{\widetilde{X}}_{\alpha}\right]^{2} d \alpha \\
& =\frac{1}{n-1} \sum_{i=1}^{n}\left[\nu\left(\widetilde{X}_{i}\right)+\int_{0}^{1} \operatorname{Var}\left(\overline{\widetilde{X}}_{\alpha}\right) d \alpha-2 \int_{0}^{1} \frac{1}{n} \operatorname{Var}\left(\widetilde{X}_{i \alpha}\right) d \alpha\right] \\
& =\frac{1}{n-1} \sum_{i=1}^{n}\left[\nu\left(\widetilde{X}_{i}\right)+\frac{1}{n} \nu\left(\widetilde{X}_{i}\right)-\frac{2}{n} \nu\left(\widetilde{X}_{i}\right)\right] \\
& =\nu(\tilde{X}) .
\end{aligned}
$$

\section{Bootstrap methods for testing hypotheses about the variance}

In the following, using the general bootstrap methodology, we follow the application of the bootstrap in fuzzy environment and propose an approach to testing hypotheses on the basis of the evidence supplied by a set of sample fuzzy data. The treatment of the bootstrap method described here comes from Efron and Tibshirani [8] (see also [31]). The interested reader is referred to that text for more information on the underlying theory behind the bootstrap.

\subsection{One-sample test of hypotheses}

In this section we describe on way to get bootstrap hypothesis testing about the variance of fuzzy random variables based on one-sample fuzzy data. Suppose that we have fuzzy random sample $\widetilde{\mathbf{X}}=\left(\widetilde{X}_{1}, \widetilde{X}_{2}, \ldots, \widetilde{X}_{n}\right)$, and we want to test the following hypotheses

$$
\begin{aligned}
& H_{0}: \text { the variance of population is } \sigma_{0}^{2}, \\
& H_{1}: \text { the variance of population is not } \sigma_{0}^{2} .
\end{aligned}
$$

The bootstrap is a method of Monte Carlo simulation where no parametric assumptions are made about the underlying population that generated the random sample $\widetilde{\mathbf{X}}=\left(\widetilde{X}_{1}, \widetilde{X}_{2}, \ldots, \widetilde{X}_{n}\right)$. Instead, we use the sample as an estimate of the population. This estimate is called the empirical distribution $F_{n}$ where each $\widetilde{X}_{i}(i=1, \ldots, n)$ has probability mass $\frac{1}{n}$. Thus, each $\widetilde{X}_{i}(i=1, \ldots, n)$ has the same likelihood of being selected in a new sample taken from $F_{n}$. When we use $F_{n}$ as our pseudo-population, then we resample with replacement from the original sample $\widetilde{\mathbf{X}}=\left(\widetilde{X}_{1}, \widetilde{X}_{2}, \ldots, \widetilde{X}_{n}\right)$. We denote the new sample obtained in this manner by $\mathbf{X}^{* b}=\left(\widetilde{X}_{1}^{* b}, \widetilde{X}_{2}^{* b}, \ldots, \widetilde{X}_{n}^{* b}\right)$. We use the notation $\widetilde{\mathbf{X}}^{* b}(b=1, \ldots, B)$ for the $b$ th bootstrap data set. These $B$ bootstrap replicates provide us with an estimate of the distribution of the test statistic which will be introduced later. But, we need a distribution that estimates the population of treatment times under $H_{0}$. Note first that the empirical distribution (i.e., putting probability $\frac{1}{n}$ on each member of $\widetilde{\mathbf{X}}$ ) is not an appropriate estimate for the population distribution because it does not obey $H_{0}$. Somehow we need to obtain an estimate of the population distribution that has variance $\sigma_{0}^{2}$. A simple way is to transfer the empirical distribution so that it has the desired variance. In other word, we use as our estimated null distribution the empirical distribution on the values

$$
\widetilde{\mathbf{X}}_{c}=\left(\frac{\sigma_{0} \widetilde{X}_{1}}{S_{n}(\widetilde{\mathbf{X}})}, \ldots, \frac{\sigma_{0} \widetilde{X}_{n}}{S_{n}(\widetilde{\mathbf{X}})}\right),
$$


because under the hypothesis $H_{0}$ one can easily show that the sample variance of $\widetilde{\mathbf{X}}_{c}$ is $\sigma_{0}^{2}$ (i.e., $\left.S_{n}^{2}\left(\widetilde{\mathbf{X}}_{c}\right)=\sigma_{0}^{2}\right)$. In testing the null hypothesis $H_{0}$ at the nominal significance level $\gamma \in[0,1]$, the following test statistic is proposed

$$
T(\widetilde{\mathbf{X}})=\frac{(n-1) S_{n}^{2}(\widetilde{\mathbf{X}})}{\sigma_{0}^{2}} .
$$

To get estimate of the distribution of the test statistic $T(\widetilde{\mathbf{X}})$ under null hypothesis $H_{0}$, we obtain $B$ bootstrap samples by sampling with replacement from the original sample $\widetilde{\mathbf{X}}_{c}=\left(\widetilde{X}_{c 1}, \ldots, \widetilde{X}_{c n}\right)$. We denote the new samples obtained in this manner by $\widetilde{\mathbf{X}}_{c}^{* 1}, \widetilde{\mathbf{X}}_{c}^{* 2}, \ldots, \widetilde{\mathbf{X}}_{c}^{* B}$. For every bootstrap sample $\widetilde{\mathbf{X}}_{c}^{* b}(b=1, \ldots, B)$, we calculate the same statistic to obtain the bootstrap replications of $T(\widetilde{\mathbf{X}})$, as follows

$$
T\left(\widetilde{\mathbf{X}}_{c}^{* b}\right)=\frac{(n-1) S_{n}^{2}\left(\widetilde{\mathbf{X}}_{c}^{* b}\right)}{\sigma_{0}^{2}}, \quad b=1, \ldots, B .
$$

Once we have the $B$ bootstrapped values $T\left(\widetilde{\mathbf{X}}_{c}^{* b}\right)$ from above equation, we can use them to understand the estimated distribution for $T(\widetilde{\mathbf{X}})$. We use the estimated distribution in the next step to estimate the quantiles needed for testing hypotheses. The $\frac{\gamma}{2}$-th quantile, denoted by $t_{\frac{\gamma}{2}}$ of the $T(\widetilde{\mathbf{X}})$, is estimated by

$$
\frac{\gamma}{2}=\frac{\text { number of }\left(T\left(\widetilde{\mathbf{X}}_{c}^{* b}\right) \leq \widehat{t}_{\frac{\gamma}{2}}\right)}{B} .
$$

We are now ready to test the hypotheses. This is given by "the null hypothesis $H_{0}$ is rejected whenever $T(\widetilde{\mathbf{X}})<\widehat{t}_{\frac{\gamma}{2}}$ or $T(\widetilde{\mathbf{X}})>\widehat{t}_{1-\frac{\gamma}{2}}$, otherwise, it is accepted".

Algorithm 1. The steps for the proposed bootstrap methodology of testing hypotheses for one-sample are given here:

1. Given a random sample $\widetilde{\mathbf{X}}=\left(\widetilde{X}_{1}, \widetilde{X}_{2}, \ldots, \widetilde{X}_{n}\right)$, calculate $\widetilde{\mathbf{X}}_{c}=\left(\widetilde{X}_{c 1}, \widetilde{X}_{c 2}, \ldots, \widetilde{X}_{c n}\right)$, and $T(\widetilde{\mathbf{X}})=\frac{(n-1) S_{n}^{2}(\widetilde{\mathbf{X}})}{\sigma_{0}^{2}}$.

2. Sample with replacement from the sample $\widetilde{\mathbf{X}}_{c}$ to get $\widetilde{\mathbf{X}}_{c}^{* 1}, \widetilde{\mathbf{X}}_{c}^{* 2}, \ldots, \widetilde{\mathbf{X}}_{c}^{* B}$.

3. Calculate the same statistic using the bootstrap samples in step 2 to get $T\left(\widetilde{\mathbf{X}}_{c}^{* b}\right)=\frac{(n-1) S_{n}^{2}\left(\widetilde{\mathbf{X}}_{c}^{* b}\right)}{\sigma_{0}^{2}}, b=$ $1, \ldots, B$.

4. Use the bootstrap replicates $T\left(\widetilde{\mathbf{X}}_{c}^{* 1}\right), T\left(\widetilde{\mathbf{X}}_{c}^{* 2}\right), \ldots, T\left(\widetilde{\mathbf{X}}_{c}^{* B}\right)$ to estimate the distribution of $T(\widetilde{\mathbf{X}})$.

5. Compute the estimated bootstrap quantiles $\widehat{t}_{\frac{\gamma}{2}}$ and $\widehat{t}_{1-\frac{\gamma}{2}}$.

6. The hypothesis $H_{0}$ is rejected whenever $T(\widetilde{\mathbf{X}})<\widehat{t}_{\frac{\gamma}{2}}$ or $T(\widetilde{\mathbf{X}})>\widehat{t}_{1-\frac{\gamma}{2}}$.

\section{Example 2}

Suppose that we have taken the fuzzy random sample given in Table 1 from a population. Based on this data set, we want to test the following hypotheses

$$
\begin{aligned}
& H_{0}: \quad \text { the variance of population is } \sigma_{0}^{2}, \\
& H_{1} \quad: \quad \text { the variance of population is not } \sigma_{0}^{2},
\end{aligned}
$$

at significance level $\gamma=0.05$ for the different values of $\sigma_{0}^{2}$ given in Table 2 .

By following the steps in Algorithm 1 with $B=10000$ bootstrap replications, one can obtain the bootstrap replicates of $T\left(\widetilde{\mathbf{x}}_{c}^{* 1}\right), T\left(\widetilde{\mathbf{x}}_{c}^{* 2}\right), \ldots, T\left(\widetilde{\mathbf{x}}_{c}^{* B}\right)$. By sorting these values we can easily estimate any $\gamma$-quantile of the estimated distribution of the test statistic $T(\widetilde{\mathbf{X}})$. This says that, for example, the estimated $\widehat{t}_{0.025}$ is the 250th largest value of the $T\left(\widetilde{\mathbf{x}}_{c}^{* b}\right)$ (because $B \times \frac{\gamma}{2}=10000 \times 0.025=250$ ), and the estimated $\widehat{t}_{0.975}$ is the 9750th largest value of the $T\left(\widetilde{\mathbf{x}}_{c}^{* b}\right)$. The estimated quantiles $\widehat{t}_{0.025}$ and $\widehat{t}_{0.975}$ for testing different values of $\sigma_{0}^{2}$ are obtained as given in Table 2. The histograms in Figure 1 show the estimated distributions of the test statistic $T(\widetilde{\mathbf{X}})$ for different values of $\sigma_{0}^{2}$ given in Table 2. Finally, at the nominal significance level 0.05 , the test result of the null hypothesis $H_{0}$ based on the fuzzy random sample given in Table 1 is given in Table 2. 
Table 1. Data set in Example 2

\begin{tabular}{|c|c|}
\hline$\widetilde{x}_{1}=(0.23,0.04,0.07)_{T}$ & $\widetilde{x}_{16}=(1.78,0.04,0.06)_{T}$ \\
\hline$\widetilde{x}_{2}=(0.76,0.05,0.02)_{T}$ & $\widetilde{x}_{17}=(1.99,0.08,0.09)_{T}$ \\
\hline$\widetilde{x}_{3}=(0.98,0.12,0.09)_{T}$ & $\widetilde{x}_{18}=(2.25,0.04,0.04)_{T}$ \\
\hline$\widetilde{x}_{4}=(1.14,0.06,0.09)_{T}$ & $\widetilde{x}_{19}=(2.45,0.01,0.08)_{T}$ \\
\hline$\widetilde{x}_{5}=(1.46,0.10,0.07)_{T}$ & $\widetilde{x}_{20}=(2.57,0.07,0.02)_{T}$ \\
\hline$\widetilde{x}_{6}=(1.69,0.05,0.12)_{T}$ & $\widetilde{x}_{21}=(0.64,0.11,0.07)_{T}$ \\
\hline$\widetilde{x}_{7}=(1.95,0.05,0.11)_{T}$ & $\widetilde{x}_{22}=(0.94,0.09,0.04)_{T}$ \\
\hline$\widetilde{x}_{8}=(2.17,0.03,0.05)_{T}$ & $\widetilde{x}_{23}=(1.08,0.10,0.06)_{T}$ \\
\hline$\widetilde{x}_{9}=(2.40,0.08,0.12)_{T}$ & $\widetilde{x}_{24}=(1.37,0.08,0.06)_{T}$ \\
\hline$\widetilde{x}_{10}=(2.51,0.10,0.14)_{T}$ & $\widetilde{x}_{25}=(1.64,0.02,0.08)_{T}$ \\
\hline$\widetilde{x}_{11}=(0.41,0.03,0.08)_{T}$ & $\widetilde{x}_{26}=(1.83,0.09,0.05)_{T}$ \\
\hline$\widetilde{x}_{12}=(0.86,0.08,0.04)_{T}$ & $\widetilde{x}_{27}=(2.04,0.11,0.06)_{T}$ \\
\hline$\widetilde{x}_{13}=(1.02,0.03,0.10)_{T}$ & $\widetilde{x}_{28}=(2.36,0.05,0.09)_{T}$ \\
\hline$\widetilde{x}_{14}=(1.23,0.03,0.14)_{T}$ & $\widetilde{x}_{29}=(2.49,0.13,0.05)_{T}$ \\
\hline$\widetilde{x}_{15}=(1.53,0.13,0.15)_{T}$ & $\widetilde{x}_{30}=(2.61,0.08,0.06)_{T}$ \\
\hline
\end{tabular}

Table 2. Test result for testing different values of $\sigma_{0}^{2}$ in Example 2 (see also Figure 1)

\begin{tabular}{|c|r|r|r|l|}
\hline$\sigma_{0}^{2}$ & $T(\widetilde{\mathbf{x}})$ & $\widehat{t}_{0.025}$ & $\widehat{t}_{0.025}$ & Result \\
\hline 0.28 & 50.61 & 18.48 & 38.51 & reject $H_{0}$ \\
\hline 0.38 & 37.20 & 18.50 & 38.51 & accept $H_{0}$ \\
\hline 0.48 & 29.58 & 18.50 & 38.51 & accept $H_{0}$ \\
\hline 0.68 & 20.81 & 18.52 & 38.51 & accept $H_{0}$ \\
\hline 0.90 & 15.73 & 18.54 & 38.54 & reject $H_{0}$ \\
\hline 1.50 & 9.36 & 18.54 & 38.54 & reject $H_{0}$ \\
\hline
\end{tabular}

\subsection{Two-sample test of hypotheses}

In this section we present a bootstrap approach to the two-sample test of equality of variances. In this way, let $\left(\Omega_{X}, \mathcal{A}_{X}, \mathbf{P}_{X}\right)$ and $\left(\Omega_{Y}, \mathcal{A}_{Y}, \mathbf{P}_{Y}\right)$ be two probability spaces, and let $\widetilde{\mathbf{X}}=\left(\widetilde{X}_{1}, \widetilde{X}_{2}, \ldots, \widetilde{X}_{n}\right)$ and $\widetilde{\mathbf{Y}}=$ $\left(\widetilde{Y}_{1}, \widetilde{Y}_{2}, \ldots, \widetilde{Y}_{m}\right)$ be two independent fuzzy random variables associated with these spaces, respectively. Also let $\sigma_{X}^{2}$ and $\sigma_{Y}^{2}$ be the corresponding population variances. The goal of this section is testing the hypotheses

$$
\begin{aligned}
& H_{0}: \text { the variances of the two populations are equal, (i.e. } \frac{\sigma_{X}^{2}}{\sigma_{Y}^{2}}=1 \text { ), } \\
& H_{1} \quad: \quad \text { the variances of the two populations are not equal, (i.e. } \frac{\sigma_{X}^{2}}{\sigma_{Y}^{2}} \neq 1 \text { ), }
\end{aligned}
$$

on the basis of the available sample fuzzy information. To get bootstrap populations with a common variance from the available sample information in this case, one can multiply to each sample $\widetilde{\mathbf{X}}$ and $\widetilde{\mathbf{Y}}$ the $\operatorname{ratios} \frac{1}{S_{n}(\widetilde{\mathbf{X}})}$ and $\frac{1}{S_{m}(\widetilde{\mathbf{Y}})}$, respectively. In other words, one can define new fuzzy random variables

$$
\begin{aligned}
& \widetilde{\mathbf{X}}_{c}=\left(\frac{\widetilde{X}_{1}}{S_{n}(\widetilde{\mathbf{X}})}, \ldots, \frac{\widetilde{X}_{n}}{S_{n}(\widetilde{\mathbf{X}})}\right) \\
& \widetilde{\mathbf{Y}}_{c}=\left(\frac{\widetilde{Y}_{1}}{S_{m}(\widetilde{\mathbf{Y}})}, \ldots, \frac{\widetilde{Y}_{m}}{S_{m}(\widetilde{\mathbf{Y}})}\right) .
\end{aligned}
$$



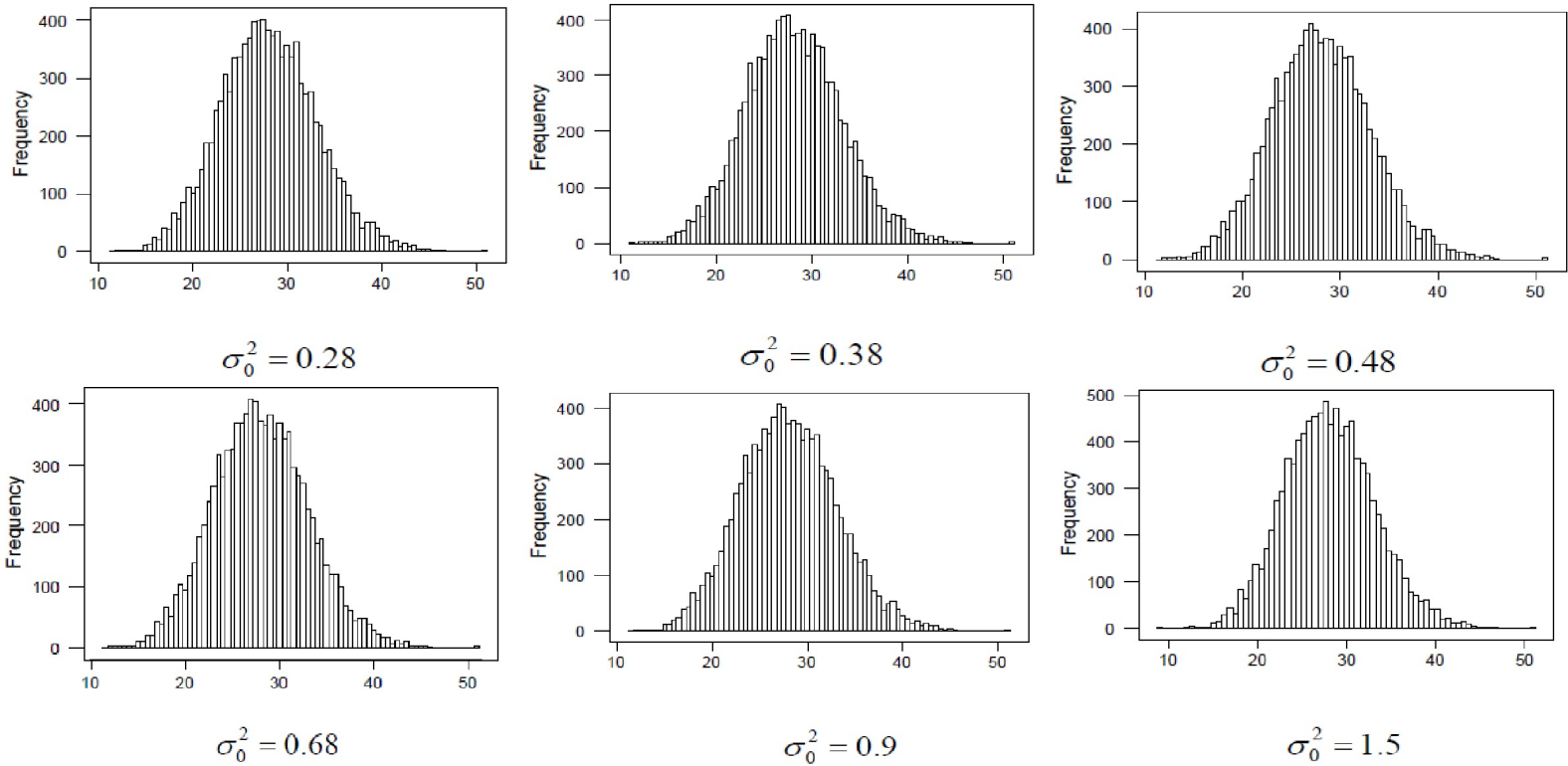

Figure 1. The histograms of the estimated distributions of the test statistic $T(\widetilde{\mathbf{X}})$ for different values of $\sigma_{0}^{2}$ given in Table 2 in Example 2.

Under the hypothesis $H_{0}$ one can easily show that the ratio of sample variances of $\widetilde{\mathbf{X}}_{c}$ and $\widetilde{\mathbf{Y}}_{c}$ is 1 (i.e., $\left.\frac{S_{n}^{2}\left(\widetilde{\mathbf{X}}_{c}\right)}{S_{m}^{2}\left(\widetilde{\mathbf{Y}}_{c}\right)}=1\right)$. Therefore, resampling from these new populations is considered. In testing the null hypothesis $H_{0}: \frac{\sigma_{X}^{2}}{\sigma_{Y}^{2}}=1$ at the nominal significance level $\gamma \in[0,1]$, the following test statistic is proposed

$$
T(\widetilde{\mathbf{X}}, \widetilde{\mathbf{Y}})=\frac{(n-1) S_{n}^{2}(\widetilde{\mathbf{X}})}{(m-1) S_{m}^{2}(\widetilde{\mathbf{Y}})}
$$

The null hypothesis $H_{0}$ should be rejected whenever $T(\widetilde{\mathbf{X}}, \widetilde{\mathbf{Y}})<t_{\frac{\gamma}{2}}$ or $T(\widetilde{\mathbf{X}}, \widetilde{\mathbf{Y}})>t_{1-\frac{\gamma}{2}}$ where $t_{\gamma}$ is the $100(1-$ $\gamma) \%$ quantile of the bootstrap distribution of the test statistic $T(\widetilde{\mathbf{X}}, \widetilde{\mathbf{Y}})$. Similar to the conclusions obtained for the one-sample test for the variance in previous section, the approach to be considered in the following will deal with the same. Therefore, to get estimate of the distribution of the test statistic $T(\widetilde{\mathbf{X}}, \widetilde{\mathbf{Y}})$ under null hypothesis $H_{0}$, we obtain $B$ bootstrap samples by sampling with replacement from the original samples $\widetilde{\mathbf{X}}_{c}$, and $\widetilde{\mathbf{Y}}_{c}$. For every bootstrap sample $\left(\widetilde{\mathbf{X}}_{c}^{* b}, \widetilde{\mathbf{Y}}_{c}^{* b}\right)(b=1, \ldots, B)$, we calculate the same statistic to obtain the bootstrap replications of $T(\widetilde{\mathbf{X}}, \widetilde{\mathbf{Y}})$, as follows

$$
T\left(\widetilde{\mathbf{X}}_{c}^{* b}, \widetilde{\mathbf{Y}}_{c}^{* b}\right)=\frac{(n-1) S_{n}^{2}\left(\widetilde{\mathbf{X}}_{c}^{* b}\right)}{(m-1) S_{m}^{2}\left(\widetilde{\mathbf{Y}}_{c}^{* b}\right)}, \quad b=1, \ldots, B
$$

Once we have the $B$ bootstrapped values $T\left(\widetilde{\mathbf{X}}_{c}^{* b}, \widetilde{\mathbf{Y}}_{c}^{* b}\right)$ from above equation, we can use them to understand the estimated distribution for $T(\widetilde{\mathbf{X}}, \widetilde{\mathbf{Y}})$. We use the estimated distribution in the next step to estimate the quantiles needed for testing hypotheses. The $\frac{\gamma}{2}$-th quantile of $T(\widetilde{\mathbf{X}}, \widetilde{\mathbf{Y}})$ under null hypothesis $H_{0}$ is estimated by

$$
\frac{\gamma}{2}=\frac{\text { number of }\left(T\left(\widetilde{\mathbf{X}}_{c}^{* b}, \widetilde{\mathbf{Y}}_{c}^{* b}\right) \leq \widehat{t}_{\frac{\gamma}{2}}\right)}{B} .
$$

Algorithm 2. The steps for the proposed bootstrap methodology of testing hypotheses for two-sample fuzzy data set are given here: 
Table 3. Fuzzy random sample of size 15 from a population in Example 3

\begin{tabular}{|c|c|}
\hline$\widetilde{y}_{1}=(2.45,0.04,0.02)_{T}$ & $\widetilde{y}_{9}=(2.00,0.08,0.07)_{T}$ \\
\hline$\widetilde{y}_{2}=(1.04,0.10,0.05)_{T}$ & $\widetilde{y}_{10}=(2.02,0.11,0.02)_{T}$ \\
\hline$\widetilde{y}_{3}=(1.09,0.04,0.01)_{T}$ & $\widetilde{y}_{11}=(1.28,0.10,0.00)_{T}$ \\
\hline$\widetilde{y}_{4}=(0.51,0.07,0.08)_{T}$ & $\widetilde{y}_{12}=(0.95,0.12,0.09)_{T}$ \\
\hline$\widetilde{y}_{5}=(2.40,0.07,0.06)_{T}$ & $\widetilde{y}_{13}=(2.04,0.01,0.04)_{T}$ \\
\hline$\widetilde{y}_{6}=(1.96,0.11,0.03)_{T}$ & $\widetilde{y}_{14}=(0.68,0.02,0.05)_{T}$ \\
\hline$\widetilde{y}_{7}=(1.34,0.01,0.08)_{T}$ & $\widetilde{y}_{15}=(0.89,0.09,0.01)_{T}$ \\
\hline$\widetilde{y}_{8}=(1.43,0.08,0.09)_{T}$ & \\
\hline
\end{tabular}

Table 4. The estimated quantiles of the bootstrap distribution of $T(\widetilde{\mathbf{X}}, \widetilde{\mathbf{Y}})$ in Example 3 (see also Figure 2)

\begin{tabular}{|l|l|l|l|l|l|l|l|l|}
\hline$\gamma$ & 0.005 & 0.025 & 0.05 & 0.10 & 0.90 & 0.95 & 0.975 & 0.995 \\
\hline$\widehat{t}_{\gamma}$ & 1.43 & 1.67 & 1.79 & 1.98 & 3.16 & 4.16 & 5.10 & 6.98 \\
\hline
\end{tabular}

1. Given two random samples $\widetilde{\mathbf{X}}=\left(\widetilde{X}_{1}, \widetilde{X}_{2}, \ldots, \widetilde{X}_{n}\right)$, and $\widetilde{\mathbf{Y}}=\left(\widetilde{Y}_{1}, \widetilde{Y}_{2}, \ldots, \widetilde{Y}_{m}\right)$, calculate

$$
\begin{aligned}
\widetilde{\mathbf{X}}_{c} & =\left(\frac{\widetilde{X}_{1}}{S_{n}(\widetilde{\mathbf{X}})}, \ldots, \frac{\widetilde{X}_{n}}{S_{n}(\widetilde{\mathbf{X}})}\right), \\
\widetilde{\mathbf{Y}}_{c} & =\left(\frac{\widetilde{Y}_{1}}{S_{m}(\widetilde{\mathbf{Y}})}, \ldots, \frac{\widetilde{Y}_{m}}{S_{m}(\widetilde{\mathbf{Y}})}\right), \\
T(\widetilde{\mathbf{X}}, \widetilde{\mathbf{Y}}) & =\frac{(n-1) S_{n}^{2}(\widetilde{\mathbf{X}})}{(m-1) S_{m}^{2}(\widetilde{\mathbf{Y}})} .
\end{aligned}
$$

2. Sample with replacement from the samples $\widetilde{\mathbf{X}}_{c}$ and $\widetilde{\mathbf{Y}}_{c}$ to get $\widetilde{\mathbf{X}}_{c}^{* 1}, \widetilde{\mathbf{X}}_{c}^{* 2}, \ldots, \widetilde{\mathbf{X}}_{c}^{* B}$, and $\widetilde{\mathbf{Y}}_{c}^{* 1}, \widetilde{\mathbf{Y}}_{c}^{* 2}, \ldots, \widetilde{\mathbf{Y}}_{c}^{* B}$.

3. Calculate the same statistic using the bootstrap samples in step 2 to get $T\left(\widetilde{\mathbf{X}}_{c}^{* b}, \widetilde{\mathbf{Y}}_{c}^{* b}\right)=\frac{(n-1) S_{n}^{2}\left(\widetilde{\mathbf{X}}_{c}^{* b}\right)}{(m-1) S_{m}^{2}\left(\widetilde{\mathbf{Y}}_{c}^{* b}\right)}$, $b=1, \ldots, B$.

4. Use the bootstrap replicates $T\left(\widetilde{\mathbf{X}}_{c}^{* 1}, \widetilde{\mathbf{Y}}_{c}^{* 1}\right), T\left(\widetilde{\mathbf{X}}_{c}^{* 2}, \widetilde{\mathbf{Y}}_{c}^{* 2}\right), \ldots, T\left(\widetilde{\mathbf{X}}_{c}^{* B}, \widetilde{\mathbf{Y}}_{c}^{* B}\right)$, to estimate the distribution of $T(\widetilde{\mathbf{X}}, \widetilde{\mathbf{Y}})$ under null hypothesis $H_{0}$.

5. Compute the estimated bootstrap quantiles $\widehat{t}_{\frac{\gamma}{2}}$ and $\widehat{t}_{1-\frac{\gamma}{2}}$.

6. The hypothesis $H_{0}$ is rejected whenever $T(\tilde{\mathbf{X}}, \widetilde{\mathbf{Y}})<\widehat{t}_{\frac{\gamma}{2}}$, or $T(\widetilde{\mathbf{X}}, \widetilde{\mathbf{Y}})>\widehat{t}_{1-\frac{\gamma}{2}}$.

\section{Example 3}

Suppose that we have taken two fuzzy random samples of size $n=30$ (Table 1) and $m=15$ (Table 3) from two populations and we want to test the equality of variances of the two populations at significance level $\gamma \in[0,1]$.

By following the steps in Algorithm 2 with $B=20000$ bootstrap replications, one can obtain the estimated quantiles of the bootstrap distribution of $T(\widetilde{\mathbf{X}}, \widetilde{\mathbf{Y}})$ under null hypothesis as given in Table 4 . Now we can easily test the hypotheses of interest at significance level $\gamma \in[0,1]$ by checking the inequalities $T(\widetilde{\mathbf{x}}, \widetilde{\mathbf{y}})<\widehat{t}_{\frac{\gamma}{2}}$, and $T(\widetilde{\mathbf{x}}, \widetilde{\mathbf{y}})>\widehat{t}_{1-\frac{\gamma}{2}}$. The histogram for the bootstrap replicates of $T\left(\widetilde{\mathbf{x}}_{c}^{* 1}, \widetilde{\mathbf{y}}_{c}^{* 1}\right), T\left(\widetilde{\mathbf{x}}_{c}^{* 2}, \widetilde{\mathbf{y}}_{c}^{* 2}\right), \ldots, T\left(\widetilde{\mathbf{x}}_{c}^{* B}, \widetilde{\mathbf{y}}_{c}^{* B}\right)$ is shown in Figure 2, showing the estimated distributions of the test statistic $T(\widetilde{\mathbf{X}}, \widetilde{\mathbf{Y}})$.

\section{Conclusions}

In this paper we considered hypothesis-tests for the (crisp-valued) variance of a fuzzy random variable in a population. For this purpose, we made use of a new definition of fuzzy random variables and a new metric for 


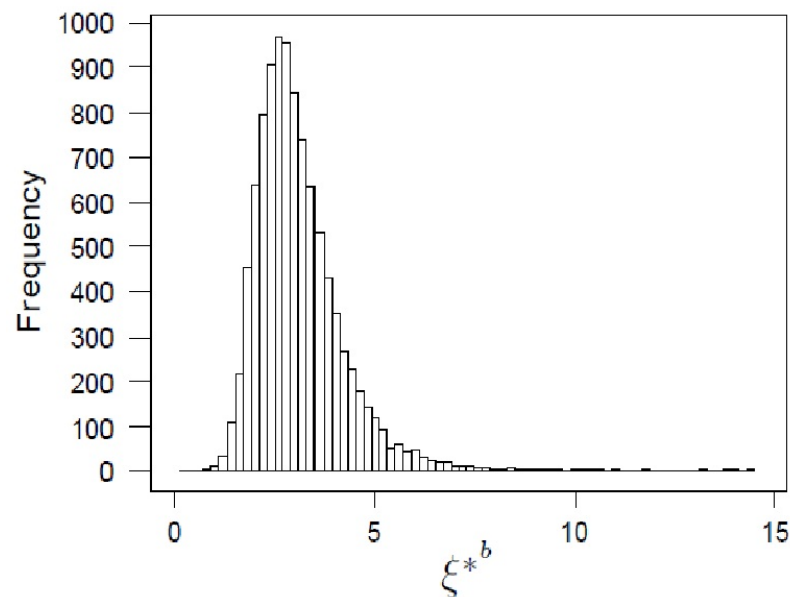

Figure 2. The estimated distributions of the test statistic $T(\widetilde{\mathbf{X}}, \widetilde{\mathbf{Y}})$ in Example 3.

fuzzy numbers. Using the general bootstrap methodology, we followed the application of the bootstrap in fuzzy environment and proposed an approach to testing hypotheses on the basis of the evidence supplied by a set of sample fuzzy data. It should be pointed out that the proposed procedure in this paper can be applied in practice with no limitations.

The distribution of the test statistic in this paper under the alternative hypothesis can be obtained by means of either asymptotic or bootstrap techniques, which seems that the study of the power function of the tests proposed in this paper can be a potential research for future studies. The bootstrap approach to test the multi-sample hypothesis of variances is another topic for further research in the future, as well as discussing the adequacy of the tests through a simulation comparative study.

\section{REFERENCES}

1. M. G. Akbari, and A. Rezaei, Bootstrap testing fuzzy hypotheses and observations on fuzzy statistic, Expert Systems with Applications, vol. 37, pp. 5782-5787, 2010.

2. K. Atanassov, Intuitionistic Fuzzy Sets: Theory and Applications, Physica-Verlag, Heidelberg, 1999.

3. R. J. Auman, Integrals of set-valued functions, Journal of Mathematical Analysis and Applications, vol. 12, pp. 1-12, 1965.

4. P. Billingsley, Probability and Measure, 3rd ed, Wiley, New York, 1995.

5. A., Blanco-Fernández, M.R., Casals, A., Colubi, N., Corral, M., García-Bárzana, M.A., Gil, G., González-Rodríguez, M.T., López, M.A., Lubiano, M., Montenegro, A.B., Ramos-Guajardo, S. De La Rosa De Sáa, and B. Sinova, Random fuzzy sets: a mathematical tool to develop statistical fuzzy data analysis, Iranian Journal of Fuzzy Systems, vol. 10, pp. 1-28, 2013.

6. J. Chachi, On distribution characteristics of a fuzzy random variable, Austrian Journal of Statistics, 2017, In Press.

7. J. Chachi, and S.M. Taheri, Optimal statistical tests based on fuzzy random variables, Iranian Journal of Fuzzy Systems, 2017, In Press.

8. B. Efron, and R. J. Tibshirani, An Introduction to the Bootstrap, Chapman and Hall, London, 1993.

9. M. Frechet, Les elements aleatoires de natures quelconque dans une space distancie, Ann. Inst. H. Poincare, vol. 10, no. 4, pp. 215-310, 1948. (In Ferench).

10. W. L. Gau, and D. J. Buehrer, Vague sets, IEEE Transactions on Systems Man and Cybernetics, vol. 23, pp. 610-614, 1993.

11. M.Á. Gil, M. Montenegro, G. González-Rodríguez, A. Colubi, and M. R. Casals, Bootstrap approach to the multi-sample test of means with imprecise data, Computational Statistics and Data Analysis, vol. 51, pp. 148-162, 2006.

12. M.Á. Gil, M. López-Díaz, and D. A. Ralescu, Overview on the development of fuzzy random variables, Fuzzy Sets and Systems, vol. 157, pp. 2546-2557, 2006.

13. G. González-Rodríguez, M. Montenegro, A. Colubi, and M. A. Gil, Bootstrap techniques and fuzzy random variables: Synergy in hypothesis testing with fuzzy data, Fuzzy Sets and Systems, vol. 157, pp. 2608-2613, 2006.

14. M. B. Gorzalzany, A method of inference in approximate reasoning based on interval-valued fuzzy sets, Fuzzy Sets and Systems, vol. 21, pp. 1-17, 1987.

15. G. Hesamian, and J. Chachi, Two-sample Kolmogorov-Smirnov fuzzy test for fuzzy random variables, Statistical Papers, vol. 56, pp. $61-82,2013$

16. R. Korner, On the variance of random fuzzy variables, Fuzzy Sets and Systems, vol. 92, no. 1, pp. 83-93, 1997. 
17. V. Krätschmer, A unified approach to fuzzy random variables, Fuzzy Sets and Systems, vol. 123, pp. 1-9, 2001.

18. R. Kruse, and K. D. Meyer, Statistics with Vague Data, Reidel Publishing Company, Dordrecht, 1987.

19. H. Kwakernaak, Fuzzy random variables part I: Definitions and theorems, Information Sciences, vol. 19, pp. 1-15, 1978.

20. H. Kwakernaak, Fuzzy random variables part II: Algorithms and examples for the discrete case, Information Sciences, vol. 17, pp. 253-278, 1979.

21. B. Liu, Theory and Practice of Uncertain Programming, Physica-Verlag, Heidelberg, 2002.

22. B. Liu, Uncertainty Theory, 5th ed, Springer-Verlag, Berlin (URL: http://orsc.edu.cn/liu/ut.pdf), 2016

23. B. Liu, and Y. K. Liu, Expected value of fuzzy variable and fuzzy expected value models, IEEE Transactions on Fuzzy Systems, vol. 10, pp. 445-450, 2002.

24. Y. K. Liu, and B. Liu, Fuzzy random variables: A scalar expected value operator, Fuzzy Optimization and Decision Making, vol. 2, pp. 143-160, 2003.

25. M. Montenegro, A. Colubi, M. R. Casals, and M.Á. Gil, Asymptotic and bootstrap techniques for testing the expected value of a fuzzy random variable, Metrika, vol. 59, pp. 31-49, 2004.

26. J. Peng, and B. Liu, Some properties of optimistic and pessimistic values of fuzzy, IEEE International Conference on Fuzzy Systems, vol. 2, pp. 745-750, 2004

27. M. L. Puri, and D. A. Ralescu, The concept of normality for fuzzy random variables, The Annals of Probability, vol. 13, pp. 1373-1379, 1985.

28. M. L. Puri, and D. A. Ralescu, D.A., Fuzzy random variables, Journal of Mathematical Analysis and Applications, vol. 114, pp. 409-422, 1986.

29. A. B. Ramos-Guajardo, A. Colubi, G. González-Rodríguez, and M. A. Gil, One sample tests for a generalized Fréchet variance of a fuzzy random variable, Metrika, vol. 71, no. 2, pp. 185-202, 2010.

30. A. B. Ramos-Guajardo, and M. A. Lubiano, $K$-sample tests for equality of variances of random fuzzy sets, Computational Statistics and Data Analysis, vol. 56, no. 4, pp. 956-966, 2012.

31. J. Shao, and D. Tu, The Jackknife and Bootstrap. Springer, NewYork, 1995.

32. S. M. Taheri, and G. Hesamian, A generalization of the Wilcoxon signed-rank test and its applications, Stat Papers, vol. 54, pp. 457-470, 2013.

33. R. Viertl, Statistical Methods for Fuzzy Data, Wiley, Chichester, 2011.

34. R. A. Vitale, An alternate formulation of mean value for random geometric figures, J. Microscopy, vol. 151, no. 3, pp. 197-204, 1998.

35. S. Yosefi, M. Arefi, and M. G. Akbari, A new approach for testing fuzzy hypotheses based on likelihood ratio statistic, Stat Papers, vol. 57, pp. 665-688, 2016.

36. L. A. Zadeh, Fuzzy sets, Information and Control, vol. 8, pp. 338-353, 1965.

37. Z. Zainali, M. G. Akbari, and H. Alizadeh Noughabi, Intuitionistic fuzzy random variable and testing hypothesis about its variance, Soft Computing, vol. 19, no. 9, pp. 2681-2689, 2015

38. H. J. Zimmermann, Fuzzy Set Theory and Its Applications, 4th ed., Kluwer Nihoff, Boston, 2001. 